\title{
Aptamer-Based Colorimetric Probe for trans-Zeatin Detection Using Unmodified Gold Nanoparticle
}

\author{
Ping Sun $\mathbb{D}^{1,2}$ Xiwei Zhang, ${ }^{3}$ and Xianxiang Wang ${ }^{3}$ \\ ${ }^{1}$ Information Materials and Device Applications Key Laboratory of Sichuan Provincial Universities, \\ Chengdu University of Information Technology, Chengdu 610225, China \\ ${ }^{2}$ State Key Laboratory of Electronic Thin Films and Integrated Devices, University of Electronic Science and Technology of China, \\ Chengdu 610054, China \\ ${ }^{3}$ College of Science, Sichuan Agricultural University, Chengdu 611130, China
}

Correspondence should be addressed to Ping Sun; sunping197725@163.com

Received 6 July 2020; Revised 15 September 2020; Accepted 15 October 2020; Published 27 October 2020

Academic Editor: Anastasios S. Economou

Copyright (c) 2020 Ping Sun et al. This is an open access article distributed under the Creative Commons Attribution License, which permits unrestricted use, distribution, and reproduction in any medium, provided the original work is properly cited.

Trans-Zeatin is the major active phytohormone in immature corn kernels. Herein, a highly sensitive, good selective and simple aptamer-based colorimetric method for the detection of trans-zeatin was constructed. The selected aptamer sequence binds with trans-zeatin and induces a duplex-to-aptamer structure switching. The gold nanoparticles (AuNPs) solution is stable with highconcentration salt, which is protected by red complementary DNA. In the absence of trans-zeatin, the color of AuNPs changed from red to blue because aptamer DNA and complementary DNA form double-stranded DNA. Thus, the ratio of absorbance intensities (A522/A650) of AuNPs is changed with the concentration of trans-zeatin. The color change could be observed by the naked eye. The linear range of this method covers a large variation of trans-zeatin concentration from 0.05 to $0.75 \mu \mathrm{M}$. The detection limit is $0.037 \mu \mathrm{M}$. Moreover, this method was applied successfully to detect trans-zeatin in real plant samples.

\section{Introduction}

Trans-Zeatin, 6-(4-hydroxy-3-methyl-2-transbutenylamin o)purine is the major active phytohormone, which is discovered in immature corn kernels from the genus Zea. As one of the plant growth hormones, it plays a key role in controlling plant development and aging, including cell division, tissue differentiation, and plant growth [1, 2]. Especially, the levels of trans-zeatin have a large effect on plants. It can promote callus initiation, retard yellowing for vegetables and fruit after blossom, and cause auxiliary stems to grow and flower [3]. Hence, it is very important to detect the content of trans-zeatin in agricultural production. The current detection methods are still largely dependent on enzyme-linked immunosorbent assay (ELISA) [4-6] or high-performance liquid chromatography (HPLC) [7, 8]. The mechanism of ELISA is using zeatin as an antigen to react with specific labeled antibody. This method is highly sensitive, but it has high cost for expensive agents and time consuming for cockamamie operation steps. Meanwhile, overall recoveries of HPLC are not even 10\% sometimes, which requires tedious preprocessing steps and expensive instrumentation [9]. It is necessary to find a simpler, more responsive, and easily operable method to measure the quantity of trans-zeatin in many agricultural fields.

Aptamers are selected in vitro through systematic evolution of ligands by exponential enrichment (SELEX). The artificial single-stranded DNA and RNA sequences that fold into secondary and tertiary structures make them bind to certain targets with extremely high specificity and affinity. They possess high recognition for small inorganic or organic substances, even proteins or cells [10-12]. Therefore, a different aptamer has been developed as a specific recognition probe for target. Many analytical methods were reported, such as fluorescence [13, 14], electrochemistry [15], chemiluminescence $[16,17]$, and colorimetric $[18,19]$. Compared with the above methods, colorimetric is a convenient alternative method which can be carried out without 
any special expensive instrumentation and some costly reagents [20-23]. Gold nanoparticles (AuNPs) have been used widely as a colorimetric probe, due to the shift of surface plasmon resonance interfered by other ions or molecules. The color of dispersed AuNPs is red, and the aggregated is blue. Aptamers are single-stranded oligonucleotides (ssDNA or ssRNA). ssDNA with random coil structures could adsorb on the surface of AuNPs and protect AuNPs from salt-induced aggregation [24]. Because of distinctive adsorptive properties of the aptamers toward AuNPs, the binding of the target or conformational changes in the DNA result in assembly of the AuNPs to produce colorimetric signal [25].

In this study, a trans-zeatin's aptamer was used to protect AuNPs against salts. The AuNPs are applied as a colorimetric probe for trans-zeatin because the color of AuNPs will be changed from blue or purple to red with the concentration of trans-zeatin. Compared with the other methods, this colorimetric method allows qualitative detection of trans-zeatin in naked eye (without the aid of any instrument) in an uncomplicated and inexpensive manner with low concentration.

\section{Experimental}

2.1. Materials and Apparatus. Trans-Zeatin, adenosine, adenine, adenosine monophosphate, adenosine diphosphate, and adenosine triphosphate were bought from SigmaAldrich. $\mathrm{HAuCl}_{4} \cdot 3 \mathrm{H}_{2} \mathrm{O}$, sodium citrate, and other chemicals were purchased from Kelong Reagent Co., Chengdu, China. All reagents were of analytical grade and used without any further purification.

The trans-zeatin's aptamer with the sequence of $5^{\prime}$ - CGG ATA TGG TTA GGC AGG CAT AAG AGG TTT ATC CG $-3^{\prime}$ and its complementary DNA with the sequence of $5^{\prime}$ CGG ATA AAC CTC TTA TGC CTG CCT AAC CAT ATC CG $-3^{\prime}$ was adopted from Qi et al.'s work [26]. They were synthesized by Sangon Biotech Co., Ltd. (Shanghai, China) and purified by the method of polyacrylamide gel electrophoresis. Di et al. have generated the aptamers against zeatin by SELEX technique and developed a simple and selective fluorescent sensor for zeatin by integrating graphene oxide (GO) [26]. The concentration of DNA was determined by measuring the UV-vis absorption at $260 \mathrm{~nm}$ with an extinction coefficient of $204,600 \mathrm{M}^{-1} \cdot \mathrm{cm}^{-1}$. All solutions were prepared with water purified by a Milli-Q Purification System (Millipore, USA). The UV-vis absorption spectra were performed with an Ultrospec 6300 pro-UV-vis spectrophotometer (Amersham Biosciences). Shimazu Prominence HPLC system (Shimadzu, Japan) was used to verify the accuracy of the method.

2.2. Preparation of AuNPs. Citrate-capped AuNPs were prepared according to the classical Frens' method [27]. In brief, $100 \mathrm{~mL}$ of $0.01 \%(\mathrm{w} / \mathrm{w}) \mathrm{HAuCl}_{4}$ solution was heated to boiling for $2 \mathrm{~min}$, and then $1 \mathrm{~mL}$ of $1 \%$ sodium citrate solution was added rapidly with vigorous stirring. The color of the mixed solution changed from pale yellow to deep red. The heating was stopped after reflux for another $15 \mathrm{~min}$.
Thereafter, the solution was cooled to room temperature while being stirred continuously. Finally, the cooled solution was diluted and stored in a refrigerator at $4^{\circ} \mathrm{C}$ for further use.

2.3. Analytical Procedures. $1 \mu \mathrm{L}$ aptamer DNA solution $(10 \mu \mathrm{M})$ was mixed with different concentrations of transzeatin for trans-zeatin determination. The mixture was vortex-mixed thoroughly and incubated for $10 \mathrm{~min}$. The trans-zeatin and aptamer would interact efficiently with each other. $1 \mu \mathrm{L}$ complementary DNA solution $(10 \mu \mathrm{M})$ was added in the mixed solution and incubated another $5 \mathrm{~min}$. Afterwards, $1 \mu \mathrm{L}$ of the prepared hybridization solution was added in $100 \mu \mathrm{L}$ of AuNPs and incubated another $10 \mathrm{~min}$. Finally, $4 \mu \mathrm{L} \mathrm{NaCl}(0.2 \mathrm{M})$ was added in the mixed solution. The ratio of absorbance intensities (A522/A650) was measured to indicate the aggregation of the AuNPs using a spectrometer after $5 \mathrm{~min}$. The control groups were measured in the same processing. All experiments were conducted at ambient temperature.

2.4. Application in Plant Samples. Leaves were obtained from 5 -week-old seedlings grown in the greenhouse and were lyophilized for $48 \mathrm{~h}$ before analyses. Trans-zeatin was extracted from $10 \mathrm{~g}$ of leaves at $-20^{\circ} \mathrm{C}$ overnight. The extraction process is as follows. $500 \mathrm{~mL}$ of $0.2 \mathrm{M}$ acetic acidmethanol $(80: 20)$ and $100 \mathrm{mg} / \mathrm{mL}$ of butylated hydroxytoluene solution was added and mixed for 24 hours in the absence of light and $-20^{\circ} \mathrm{C}$. The extracts were filtered, centrifuged, and concentrated. Then, the $\mathrm{pH}$ of the extract was adjusted to 3 with $1 \mathrm{M}$ sulfuric acid. The pigments were removed with ethyl acetate. Liquid exacts were purified with syringe filters and stored in the refrigerator for future use.

To compare the accuracy of this method, samples were tested by high-performance liquid chromatography (HPLC) with UV detector. The HPLC is equipped with a C18 $250 \mathrm{~mm} \times 4.6 \mathrm{~mm}(5 \mu \mathrm{m})$ ODS- 2 column. The mobile phase contains methanol-water $(40: 60)$ and acidified (5\% acetic acid). The flow rate was set at $0.8 \mathrm{~mL} \cdot \mathrm{min}^{-1}$. Wavelength is $280 \mathrm{~nm}$, and analysis time is $10 \mathrm{~min}$. After establishing a standard curve with standard trans-zeatin, samples were measured, and the method was verified by standard addition method.

\section{Results and Discussion}

AuNPs's size-dependent surface resonance (SPR) absorption located at about $518 \mathrm{~nm}$ has been employed widely as a colorimetric sensor for many molecules [24, 28, 29]. The color of the gold solution reflects the degree of aggregation of AuNPs in suspension. The aggregation can be induced easily by salts. Due to the intermolecular forces and hydrophobic forces between the unmodified AuNPs and ssDNA, the strong interaction keeps them to combine tightly with each other to form an AuNPs-ssDNA conformation. The AuNPs are protected from aggregation in high-concentration salts. Figure 1 shows the detection strategy for trans-zeatin with AuNPs. In the presence of trans-zeatin, the aptamer preferentially binds to trans-zeatin and assembles a rigid duplex, 


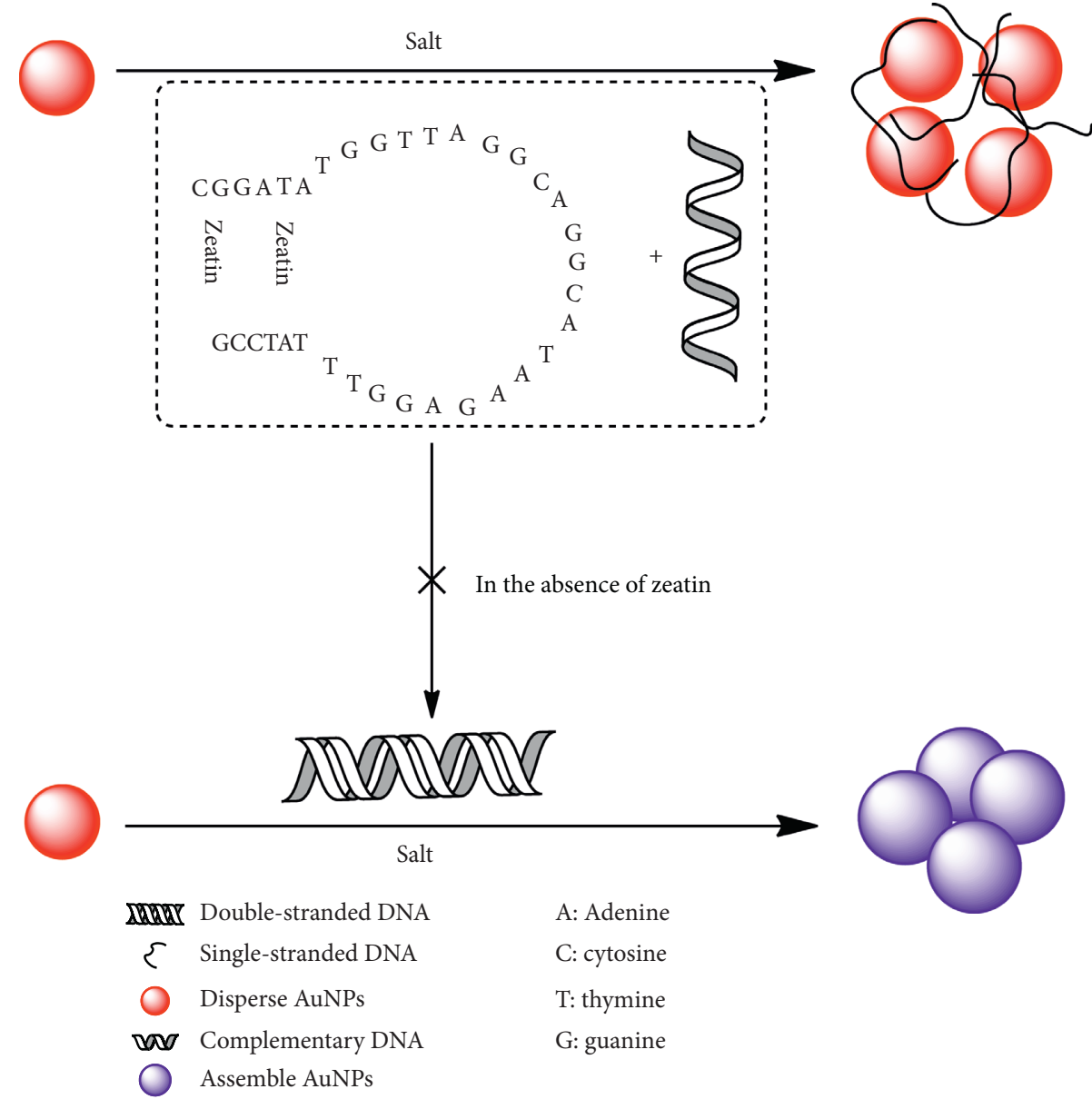

FIGURE 1: Schematic description of the AuNPs-aptamer-based strategy for trans-zeatin assay. In the presence of trans-zeatin, the binding of trans-zeatin to the aptamer sequence induces a duplex-to-aptamer structural switching. The AuNPs are stabilized by only complementary DNA, showing high resistance to salt-induced aggregation (solution staying in red). In the absence of trans-zeatin, aptamer DNA, and complementary DNA formed double-stranded DNA. AuNPs are not stabilized, and thus they are readily aggregated by salt (solution displaying blue colors).

which leads to the added ssDNA strand (complementary DNA) release. The ssDNA spontaneously binds to unmodified AuNPs through interactions and stabilizes AuNPs effectively. So, the ssDNA-stabilized AuNPs show enhanced significant resistance to salt-induced aggregation (the color of solution is still red). Otherwise, in the absence of transzeatin, double-stranded DNA will form between aptamer and its complementary DNA. As a consequence, the AuNPs is not to be protected, and thus they are readily aggregated by salt (the color displaying from red to blue). Furthermore, the changes in the dispersity of AuNPs were investigated. As shown in Figure 2, compared with AuNPs, the large aggregate was observed for the AuNPs in the absence of transzeatin, which resulted in the color change of AuNPs from wine red to blue. By adjusting the relative amount of transzeatin, we can control the color of AuNPs solution. Therefore, a colorimetric detection method for trans-zeatin was established.

In this strategy, the aptamer interacted with trans-zeatin or complementary DNA to assemble a rigid duplex due to its positive charge. That is to say, there is a competition between trans-zeatin and complementary DNA with aptamer. If the aptamer prior binds with trans-zeatin, the rest complementary DNA will spontaneously bind to unmodified AuNPs and stabilize AuNPs effectively. So, it is very important to control the relative concentration of aptamer and trans-zeatin. Figure 3 shows the effects of aptamer concentration on trans-zeatin detection. When various concentrations of aptamer between $0.5 \mu \mathrm{M}$ and $2.5 \mu \mathrm{M}$ were added into the mixed solution, the color changed, and the ratio of absorbance intensities (A522/A650) were recorded. When the aptamer is $0.5 \mu \mathrm{M}$, there is a good linear relationship between the ratios of absorbance intensities with various trans-zeatin concentrations. But the trend is more significant when the aptamer is $1 \mu \mathrm{M}$, and it is easier to observe the color change of AuNPs. Therefore, $1 \mu \mathrm{M}$ aptamer was selected for the following research.

Meanwhile, the optimization of the ratio between aptamer and complementary DNA was performed. The studies revealed that the DNA could fold into an unexpected structure due to temperature, $\mathrm{pH}$, pressure, light, and so on. And the aptamer DNA without correct 3D structure could not combine with trans-zeatin. Besides, the free end of unexpected structure effected the aggregation of AuNPs. But 


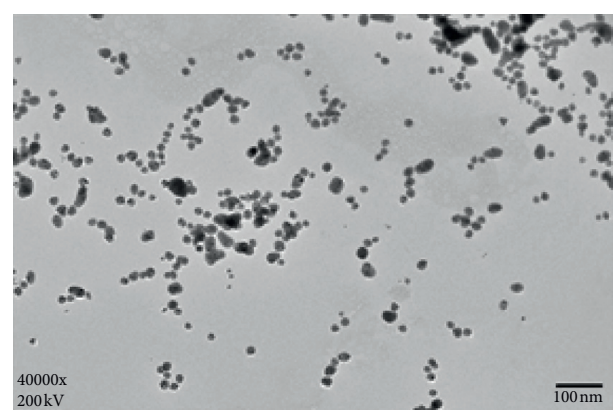

(a)

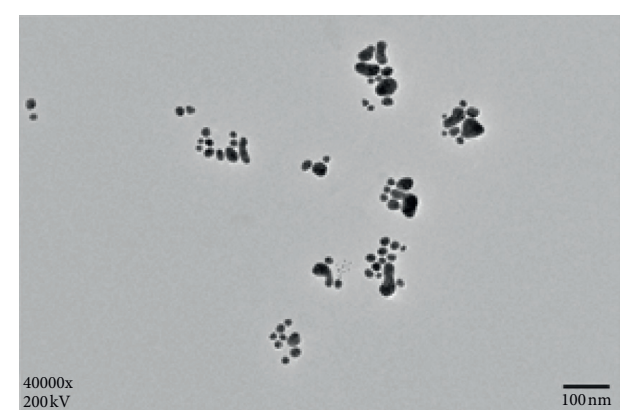

(b)

Figure 2: (a) The TEM images of AuNPs and (b) the AuNPs aggregate in the absence of a low concentration of trans-zeatin.

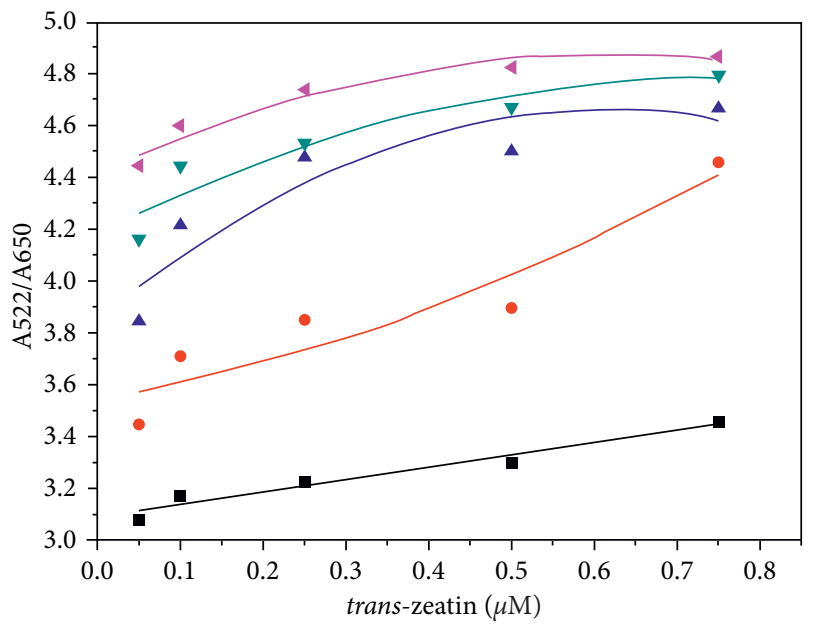

Aptamer

- $0.5 \mu \mathrm{M}$

- $1 \mu \mathrm{M}$

- $1.5 \mu \mathrm{M}$

จ $2 \mu \mathrm{M}$

$\triangleleft 2.5 \mu \mathrm{M}$

Figure 3: The effects of aptamer concentration on trans-zeatin detection. The concentrations of aptamer were $0.5,1,1.5,2$, and $2.5 \mu \mathrm{M}$, respectively.

the complementary DNA could reduce the affection significantly due to the complementary DNA hybridizing with aptamer DNA and the hybridization between complementary DNA and the end of uncorrected folded aptamer. Furthermore, an improvement of salt tolerance of AuNPs was caused by the protection of complementary ssDNA under the high concentration of trans-zeatin which was combined with aptamer DNA. So, the complementary DNA can increase significantly the reliability and sensitivity of the detection. The results revealed that either higher or lower concentrations of complementary DNA than aptamer will induce aggregation of AuNPs. Therefore, the concentration of complementary DNA was as same as the aptamer $(1 \mu \mathrm{M})$ in the study.

To detect trans-zeatin, absorption ratio of the mixed solution was measured with different concentrations of trans-zeatin under optimized conditions. Figure 4 indicated that the ratio of absorbance intensities increased with the addition of trans-zeatin. There is a very good linear relationship between absorption ratio (A522/A650) and the trans-zeatin concentration. The correlation coefficient $\left(R^{2}\right)$ is 0.98 , and the detection range is from 0.05 to $0.75 \mu \mathrm{M}$. The developed sensor has a limit of detection (LOD) which is $0.037 \mu \mathrm{M}$, which was calculated according to the $3 \delta$ criteria. Compared with reported methods for trans-zeatin detection by Di et al. (the reported LOD was $0.1 \mu \mathrm{M}$ ) [26], this method has more sensitivity for quantitative analysis of trans-zeatin. Furthermore, the color of aptamer-AuNP aggregation could be easily observed by the naked eyes when the concentration of trans-zeatin decreased. The color changed from bluepurple to wine-red (see the inset photographic images in Figure 3).

The specificity of this method was determined by challenging it with five similar molecules, including $0.75 \mu \mathrm{M}$ adenosine, adenine, adenosine monophosphate (AMP), adenosine diphosphate (ADP), and adenosine triphosphate (ATP). As shown in Figure 5, the ratio absorption (A522/ A650) for the different mixed solutions other than transzeatin had a little effect. This was due to the weak binding affinity of the aptamer-AuNPs to these molecules and little tendency to form a G-quadruplex. This implied that the proposed colorimetric method is specific for the detection of trans-zeatin.

The developed trans-zeatin colorimetric sensor based on aptamer-AuNPs was preliminarily applied to the detection of trans-zeatin in the leaves of maize. The analysis results are compared with the standard method by HPLC, which are summarized in Table 1 . The results show that the recoveries of added trans-zeatin in the samples ranged from $98.6 \%$ to $112.0 \%$ by this method, and the results agree well with standard methods by HPLC. These results demonstrate the potential applicability of this method for the quantitative detection of trans-zeatin in the real samples.

Table 2 shows a comparison of this work with those of other zeatin determination reported in the literature. The sensitivity and linear range of this work were not better than those reported in the literature, but its selectivity and accuracy were high. The newly developed colorimetric method is suitable for routine measurement of trans-zeatin. 


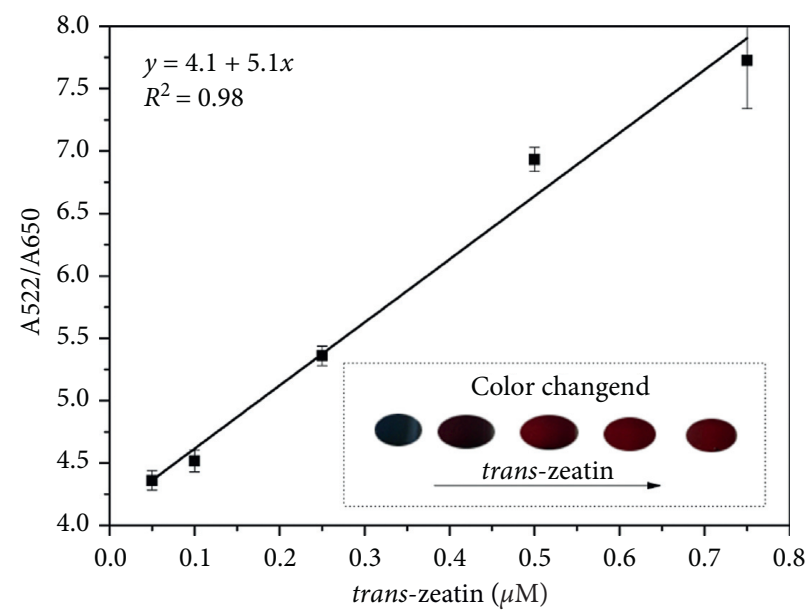

FIGURE 4: Absorption ratios (A522/A650) as a function of trans-zeatin concentration (inset: photographic images with the corresponding solutions).

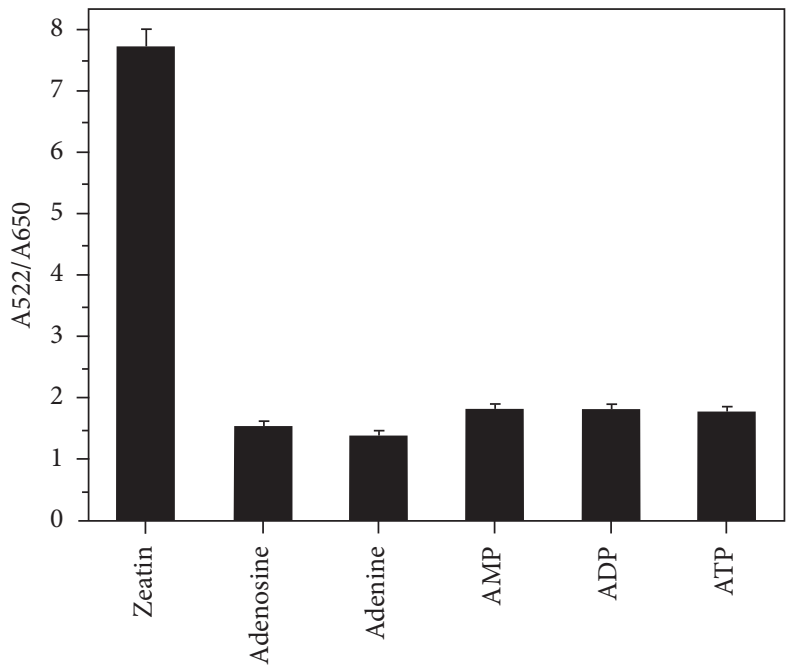

Figure 5: The relative response of the sensing system to different molecules.

TABLe 1: Analytical results for trans-zeatin in real samples.

\begin{tabular}{|c|c|c|c|c|c|}
\hline Sample & Found origin trans-zeatin $(\mu \mathrm{M})$ & Added trans-zeatin $(\mu \mathrm{M})$ & Found total trans-zeatin $(\mu \mathrm{M})$ & Recoveries (\%) & $\operatorname{RSD}(\%, n=3)$ \\
\hline \multirow[b]{2}{*}{1} & $3.221^{\mathrm{a}}$ & \multirow{2}{*}{0.10} & $3.327^{\mathrm{a}}$ & $106.0^{\mathrm{a}}$ & $0.79^{\mathrm{a}}$ \\
\hline & $3.219^{\mathrm{b}}$ & & $3.230^{\mathrm{b}}$ & $110.0^{\mathrm{b}}$ & $0.54^{\mathrm{b}}$ \\
\hline \multirow{2}{*}{2} & $3.240^{\mathrm{a}}$ & \multirow{2}{*}{0.20} & $3.464^{\mathrm{a}}$ & $112.0^{\mathrm{a}}$ & $0.65^{\mathrm{a}}$ \\
\hline & $3.236^{\mathrm{b}}$ & & $3.433^{\mathrm{b}}$ & $98.5^{\mathrm{b}}$ & $0.51^{\mathrm{b}}$ \\
\hline \multirow{2}{*}{3} & $3.302^{\mathrm{a}}$ & \multirow{2}{*}{0.30} & $3.608^{\mathrm{a}}$ & $102.0^{\mathrm{a}}$ & $0.601^{\mathrm{a}}$ \\
\hline & $3.299^{\mathrm{b}}$ & & $3.601^{\mathrm{b}}$ & $100.1^{\mathrm{b}}$ & $0.55^{\mathrm{b}}$ \\
\hline \multirow{2}{*}{4} & $3.316^{\mathrm{a}}$ & \multirow{2}{*}{0.40} & $3.711^{\mathrm{a}}$ & $98.6^{\mathrm{a}}$ & $0.72^{\mathrm{a}}$ \\
\hline & $3.312^{\mathrm{b}}$ & & $3.709^{\mathrm{b}}$ & $99.3^{\mathrm{b}}$ & $0.52^{\mathrm{b}}$ \\
\hline
\end{tabular}

${ }^{\mathrm{a}}$ Sample was measured by this method. ${ }^{\mathrm{b}}$ Sample was measured by HPLC. 
TABle 2: Comparison of some analytical methods used for the determination of zeatin.

\begin{tabular}{|c|c|c|c|}
\hline Method & Detection limit & Linear range & Reference \\
\hline Electrochemical aptasensor & $16.6 \mathrm{pM}$ & $50 \mathrm{pM}-50 \mathrm{nM}$ & {$[30]$} \\
\hline Photoelectrochemical apta-biosensor & $0.031 \mathrm{nM}$ & $0.1 \mathrm{nM}-100 \mathrm{nM}$ & {$[31]$} \\
\hline Graphene oxide-protected aptamers & $60 \mathrm{nM}$ & $0.67-6.0 \mu \mathrm{M}$ & {$[32]$} \\
\hline A hairpin aptamer and fluorescence method & $135 \mathrm{nM}$ & - & [33] \\
\hline Aptamer-based colorimetric method & $0.037 \mu M$ & $0.05-0.75 \mu \mathrm{M}$ & This work \\
\hline
\end{tabular}

\section{Conclusions}

This work developed a very simple method for the colorimetric detection of trans-zeatin in plants. The sensing mechanism is based on the increased stability of AuNPs while binding with released complementary DNA under high concentration of salts. The optical properties of AuNPs are highly distance-dependent, which leads to the direct color change observed by naked eyes. The analytical results showed that the detection limit is $0.037 \mu \mathrm{M}$ and exhibit good linear relationship from 0.05 to $0.75 \mu \mathrm{M}$. Meanwhile, this method exhibits excellent specificity and applicability. The developed method did not utilize any sample pretreatment, organic solvents, enzymatic reactions, or sophisticated instruments, thus overcoming some limitations of more conventional methods.

\section{Data Availability}

The testing data used to support the findings of this study are available from the corresponding author upon request.

\section{Conflicts of Interest}

The authors declare that they have no conflicts of interest.

\section{Acknowledgments}

This work was financially supported by the National Natural Science Foundation of China (nos. 21305097 and 61701050), project of Sichuan Provincial Department of Education (no. 2018Z073), and the Open Foundation of State Key Laboratory of Electronic Thin Films and Integrated Devices (no. KFJJ201807).

\section{References}

[1] C. O. Miller, "Evidence for the natural occurrence of zeatin and derivatives: compounds from maize which promote cell division," Proceedings of the National Academy of Sciences, vol. 54, no. 4, Article ID 1052, 1965.

[2] J. B. Cooper and S. R. Long, "Morphogenetic rescue of rhizobium meliloti nodulation mutants by trans-zeatin secretion," The Plant Cell, vol. 6, no. 2, pp. 215-225, 1994.

[3] R. A. King and J. Vanstaden, "Differential responses of buds along the shoot of Pisum-sativum to isopentenyladenine and zeatin application," Plant Physiology and Biochemistry, vol. 26, pp. 253-259, 1988.

[4] E. W. Weiler, "Radioimmunoassays for trans-zeatin and related cytokinins," Planta, vol. 149, no. 2, pp. 155-162, 1980.

[5] E. J. Trione, B. B. Krygier, G. M. Banowetz, and J. M. Kathrein, "The development of monoclonal antibodies against the cytokinin zeatin riboside," Journal of Plant Growth Regulation, vol. 4, no. 1-4, pp. 101-109, 1985.

[6] R. Maldiney, B. Leroux, I. Sabbagh, B. Sotta, L. Sossountzov, and E. Miginiac, "A biotin-avidin-based enzyme immunoassay to quantify three phytohormones: auxin, abscisic acid and zeatin-riboside," Journal of Immunological Methods, vol. 90, no. 2, pp. 151-158, 1986.

[7] Y. Matsuzaki, K. Yanagisawa, and Y. Nakamura, "Determination of zeatin and 6-benzylaminopurine in culture medium by HPLC," Bunseki Kagaku, vol. 39, no. 8, pp. T117-T120, 1990.

[8] E. A. Stahly and D. A. Buchanan, "High-performance liquid chromatographic procedure for separation and quantification of zeatin and zeatin riboside from pears, peaches and apples," Journal of Chromatography A, vol. 235, no. 2, pp. 453-459, 1982.

[9] S. P. Monselise, A. Varga, E. Knegt, and J. Bruinsma, "Course of the zeatin content in tomato fruits and seeds developing on intact or partially defoliated plants," Zeitschrift für Pflanzenphysiologie, vol. 90, no. 5, pp. 451-460, 1978.

[10] J. Yan, H. Xiong, S. Cai et al., "Advances in aptamer screening technologies,” Talanta, vol. 200, pp. 124-144, 2019.

[11] K. Y. Goud, K. K. Reddy, M. Satyanarayana, S. Kummari, and K. V. Gobi, "A review on recent developments in optical and electrochemical aptamer-based assays for mycotoxins using advanced nanomaterials," Mikrochim Acta, vol. 187, no. 1, p. 29, 2019.

[12] L. Wang, A. Wu, and G. Wei, "Graphene-based aptasensors: from molecule-interface interactions to sensor design and biomedical diagnostics," The Analyst, vol. 143, no. 7, pp. 1526-1543, 2018.

[13] X. Zeng, X. Zhang, W. Yang, H. Jia, and Y. Li, "Fluorescence detection of adenosine triphosphate through an aptamermolecular beacon multiple probe," Analytical Biochemistry, vol. 424, no. 1, pp. 8-11, 2012.

[14] S. Niu, L. Qu, Q. Zhang, and J. Lin, "Fluorescence detection of thrombin using autocatalytic strand displacement cycle reaction and a dual-aptamer DNA sandwich assay," Analytical Biochemistry, vol. 421, no. 2, pp. 362-367, 2012.

[15] Y. Wang, L. Yao, G. Ning et al., "An electrochemical strategy for tetracycline detection coupled triple helix aptamer probe with catalyzed hairpin assembly signal amplification," Biosensors and Bioelectronics, vol. 143, Article ID 111613, 2019.

[16] Y. Sun, Y. Lin, W. Sun et al., "A highly selective and sensitive detection of insulin with chemiluminescence biosensor based on aptamer and oligonucleotide-AuNPs functionalized nanosilica @ graphene oxide aerogel," Analytica Chimica Acta, vol. 1089, pp. 152-164, 2019.

[17] Y. Sun, R. Han, Y. Dai et al., "Highly selective and sensitive streptomycin chemiluminescence sensor based on aptamer and G-quadruplex DNAzyme modified three-dimensional graphene composite," Sensors and Actuators B-Chemical, vol. 301, 2019.

[18] Y. Tian, Y. Wang, Z. Sheng, T. Li, and X. Li, "A colorimetric detection method of pesticide acetamiprid by fine-tuning 
aptamer length," Analytical Biochemistry, vol. 513, pp. 87-92, 2016.

[19] J. H. Park, Y. S. Cho, S. Kang, E. J. Lee, G.-H. Lee, and S. S. Hah, "A colorimetric sandwich-type assay for sensitive thrombin detection based on enzyme-linked aptamer assay," Analytical Biochemistry, vol. 462, pp. 10-12, 2014.

[20] V. Ranganathan, S. Srinivasan, A. Singh, and M. C. DeRosa, "An aptamer-based colorimetric lateral flow assay for the detection of human epidermal growth factor receptor 2 (HER2)," Analytical Biochemistry, vol. 588, Article ID 113471, 2020.

[21] M. A. LeVatte, M. Lipfert, J. Zheng, and D. S. Wishart, "A fast, sensitive, single-step colorimetric dipstick assay for quantifying ascorbic acid in urine," Analytical Biochemistry, vol. 580, pp. 1-13, 2019.

[22] H. Tavallali, G. Deilamy-Rad, M. A. Karimi, and E. Rahimy, "A novel dye-based colorimetric chemosensors for sequential detection of $\mathrm{Cu}^{2+}$ and cysteine in aqueous solution," Analytical Biochemistry, vol. 583, Article ID 113376, 2019.

[23] B. Liu, J. Zhuang, and G. Wei, "Recent advances in the design of colorimetric sensors for environmental monitoring," Environmental Science: Nano, vol. 7, no. 8, pp. 2195-2213, 2020.

[24] Y. Ou, X. Jin, J. Liu, Y. Tian, and N. Zhou, "Visual detection of kanamycin with DNA-functionalized gold nanoparticles probe in aptamer-based strip biosensor," Analytical Biochemistry, vol. 587, Article ID 113432, 2019.

[25] A. B. Iliuk, L. Hu, and W. A. Tao, "Aptamer in bioanalytical applications," Analytical Chemistry, vol. 83, no. 12, pp. 4440-4452, 2011.

[26] C. Qi, T. Bing, H. Mei, X. Yang, X. Liu, and D. Shangguan, "Gquadruplex DNA aptamers for zeatin recognizing," Biosensors and Bioelectronics, vol. 41, pp. 157-162, 2013.

[27] G. Frens, "Controlled nucleation for the regulation of the particle size in monodisperse gold suspensions," Nature Physical Science, vol. 241, no. 105, pp. 20-22, 1973.

[28] G. Du, L. Wang, D. Zhang et al., "Colorimetric aptasensor for progesterone detection based on surfactant-induced aggregation of gold nanoparticles," Analytical Biochemistry, vol. 514, pp. 2-7, 2016.

[29] L. Liu, X. Wang, J. Yang, and Y. Bai, "Colorimetric sensing of selenocystine using gold nanoparticles," Analytical Biochemistry, vol. 535, pp. 19-24, 2017.

[30] Y. Zhou, H. Yin, Y. Wang, C. Sui, M. Wang, and S. Ai, "Electrochemical aptasensors for zeatin detection based on $\mathrm{MoS}_{2}$ nanosheets and enzymatic signal amplification," The Analyst, vol. 143, no. 21, pp. 5185-5190, 2018.

[31] Y. Wang, Y. Zhou, L. Xu, Z. Han, H. Yin, and S. Ai, "Photoelectrochemical apta-biosensor for zeatin detection based on graphene quantum dots improved photoactivity of graphite-like carbon nitride and streptavidin induced signal inhibition," Sensors and Actuators B: Chemical, vol. 257, pp. 237-244, 2018.

[32] C. Su, C. Liu, J. Chen, Z. Chen, and Z. He, "Simultaneous determination of zeatin and systemin by coupling graphene oxide-protected aptamers with catalytic recycling of DNase I," Sensors and Actuators B: Chemical, vol. 230, pp. 442-448, 2016.

[33] C. Liu, J. Chen, G. Mao, C. Su, X. Ji, and Z. He, "Targetinduced structure switching of a hairpin aptamer for the fluorescence detection of zeatin," Analytical Methods, vol. 8, no. 30, pp. 5957-5961, 2016. 